\title{
Beeinflusst der Zeitpunkt des operativen Debridements die Infektionsrate?
}

Bestehende Leitlinien empfehlen eine Notversorgung offener Frakturen mit chirurgischem Debridement innerhalb von sechs Stunden nach Trauma. Ziel der Studie von Schenker et al. war es, systematisch den Zusammenhang zwischen dem Zeitpunkt des operativen Eingriffs und konsekutiv auftretenden Infektionen zu ermitteln.

Schenker ML et al. Does timing to operative debridement affect infectious complications in open long-bone fractures? A systematic review. J Bone Joint Surg Am 2012; 94: 1057-1064

\section{Methodik \\ $\nabla$}

Die Autoren durchsuchten für ihre Studie die literarischen Datenbanken von MEDLINE, EMBASE und Cochrane sowie bestehende bibliothekarische Bestände. Dabei schlossen sie randomisierte Versuche und Kohortenstudien (retro- und prospektiv), die den Zusammenhang zwischen der Zeit bis zur operativen Versorgung und Infektionen bei offenen Frakturen beleuchteten, ein. Beschreibende oder mengenbezogene Angaben wurden nicht in die Untersuchung mit aufgenommen. Mit Hilfe eines „random effects model“ wurde daraufhin eine Meta-Analyse durchgeführt.

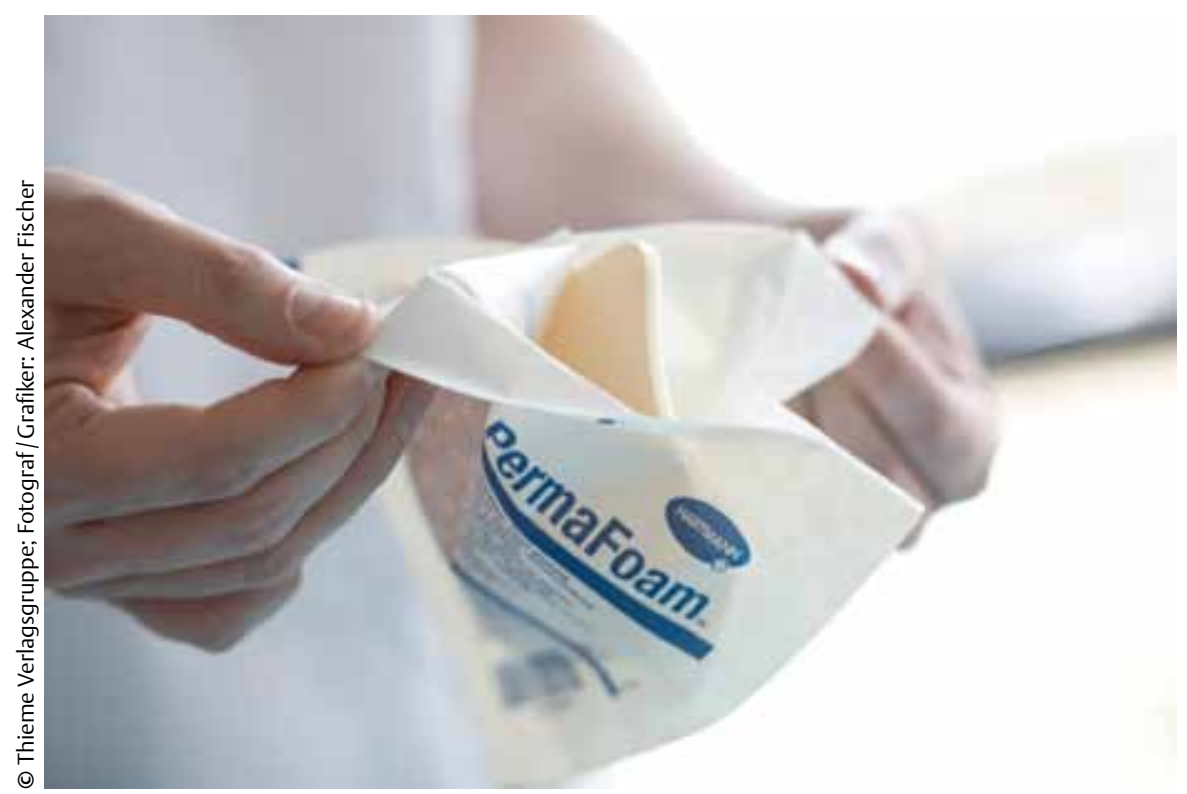

Weiterhin zweigte sich bei näherer Betrachtung der einzelnen Untergruppen kein signifikanter Unterschied. Die einzelnen Untergruppen waren hier klassifiziert nach der Verletzungsschwere (GustiloAnderson), Infektionstiefe oder Lokalisation bzw. dem Evidenzgrad der Studien.

\section{Kommentar \\ $\nabla$}

Das Zeitmanagement bei der Behandlung der offenen Frakturen erscheint uns nach heutigem Wissensstand eigentlich geklärt (Versorgung und Debridement so schnell wie möglich). So mutet die bestehende Fragestellung eher einem „alten Hut“ an als einem drängenden aktuellen Problem.

Dennoch zeigt sich insbesondere anhand der vorliegenden Meta-Analyse, dass gerade einmal 16 Studien von über $800 \mathrm{Li}$ teraturhinweisen verwertbare Informationen liefern. Ein in der offenen Frakturversorgung bestehendes „Gesetz“ der „6-Stunden-Regel“, die bereits im späten 19. Jahrhundert aufgestellt wurde, erscheint letztlich als unbewiesen. se. Davon wurden 173 Beiträge einer näheren Betrachtung hinsichtlich der Fragestellung unterzogen. Aus diesen wiederum konnten lediglich 16 eingeschlossen werden.

Zwischen einem frühen und späten Debridement offener Frakturen fand sich kein signifikanter Unterschied bezüglich auftretender Infektionen. Diese Angaben beziehen sich auf die vorliegenden Grenzwerte der eingeschlossenen Untersuchungen.

Die von den Autoren geforderten weiterführenden Studien sind notwendig. Insbesondere betrifft dies die modifizierbaren Risikofaktoren, etwa den Zeitpunkt und die Qualität des primären Managements. Doch dies ist kein triviales Unterfangen aufgrund der Vielzahl von Parametern und der vielschichtigen Effekte auf die Infektionsrate offener Frakturen.

Aus diesem Grund tun wir gut daran, uns derzeit weiterhin an der 6-Stunden-Regel zu orientieren und potenzielle Zeitverluste bis zur Primärbehandlung offener Frakturen zu minimieren.

Nadja Mielsch

Universitätsmedizin Rostock Chirurgische Klinik und Poliklinik Abt. für Unfall-, Hand- und Wiederherstellungschirurgie mielschn@med.uni-rostock.de 